\title{
Proceeding
}

Supplementary Issue: Summer Conferences of Sports Science. Costa Blanca Sports Science Events, 25-26 September 2020. Alicante, Spain.

\section{Realization of step exercises using the independent calorimetry during the period of self-isolation}

\author{
ROMAN SERGEEVICH NAGOVITSYN ${ }^{1} \triangle$, ALEKSANDER YURIEVICH OSIPOV2,3,4, MIKHAIL \\ DMITRIEVICH KUDRYAVTSEV2,4,5, SERGEY ANATOLEVICH DOROSHENKO², ANNA VLADIMIROVNA \\ VAPAEVA ${ }^{2}$ \\ ${ }^{1}$ Department of Physical Culture, Methodology and Sports, Glazov State Pedagogical Institute, Russian Federation \\ 2Department of Physical Culture, Siberian Federal University, Russian Federation \\ ${ }^{3}$ Krasnoyarsk State Medical University named after professor V.F. Voyno-Yasenetsky, Russian Federation \\ ${ }^{4}$ Siberian Law Institute of the Ministry of Internal Affair of Russia, Russian Federation \\ ${ }^{5}$ Reshetnev Siberian State University Science and Technology, Russian Federation
}

\begin{abstract}
Background: In home conditions population of Russian Federation do not have an opportunity to do optimal physical activities during the period of spreading COVID-19. The aim of the study was to develop a system of realization step exercises in home conditions using the independent calorimetry during the period regime of selfisolation and to prove its effectiveness on the indicators of body mass index of the people. Study participants: 62 women and men between the ages of 18 and 45 who have problems with being overweight. Interventions: The study was being conducted in home conditions during 60 days. During the experiment the participants independently carried out the calorimetry, taking into account the consumed calories during the day and burning kilocalories while doing step exercises and another physical activity. For carrying out the calorimetry during the day a formula was developed due to available and simple counting: « $N=0.00168-0.098 / P »$, where $N$ - the amount of burning kilocalories per one heartbeat per one $\mathrm{kg}$ of body mass, $\mathrm{P}$ - heart rate per minute. Main outcome measures: It is defined out that the introduction of step exercises in home conditions with the use of independent calorimetry to the author's system has made a significant impact $(p<.05)$ on body mass index of participants. Findings. In the presented study, for the first time, the regularities of increasing the burning of kilocalories in people at various levels of intensity depending on their body weight were experimentally revealed.

Keywords: Physical activity; Kilocalories; Home conditions; Body mass index; Self-isolation.
\end{abstract}

\section{Cite this article as:}

Nagovitsyn, R.S., Osipov, A.Y., Kudryavtsev, M.D., Doroshenko, S.A., \& Vapaeva, A.V. (2020). Realization of step exercises using the independent calorimetry during the period of self-isolation. Journal of Human Sport and Exercise, 15(4proc), S1020-S1029. doi:https://doi.org/10.14198//hse.2020.15.Proc4.04

Corresponding author. Department of Physical Culture, Methodology and Sports, Glazov State Pedagogical Institute, Russian Federation. http://orcid.org/0000-0003-4471-0875

E-mail: gto18@mail.ru

Abstract submitted to: Summer Conferences of Sports Science. Costa Blanca Sports Science Events, 25-26 September 2020. Alicante, Spain.

JOURNAL OF HUMAN SPORT \& EXERCISE ISSN 1988-5202

(C) Faculty of Education. University of Alicante

doi:10.14198/jhse.2020.15.Proc4.04

S1020 | 2020| Proc4 | VOLUME 15

(c) 2020 University of Alicante 


\section{INTRODUCTION}

Recently, many researchers have proved that the implementation of the optimal motor activity of a person in its various forms has a positive effect on the state of health, the level of physical fitness and the functional state of the body (Kraus et al., 2019). The population of various ages is offered a variety of systems for the implementation of independent and group physical exercises (Plotnikoff \& Karunamuni, 2011). Each type of motor activity is accompanied by mobile systems for monitoring and self-monitoring the state of the body during physical exertion (Henriksen et al., 2018; Wennman et al., 2019). Various programs and techniques for monitoring both individual systems, for example, cardiovascular (Abbasi, 2019; Schorr et al., 2018; Thijs et al., 2019), and all body systems (Yagotin et al., 2019) are popularized.

One of the main conditions for the full functioning of the human cardiovascular system are systematic physical exercises (Troiano et al., 2012; Westerterp \& Plasqui, 2004). It is the sequence of performing physical activity in daily, weekly and monthly modes that ensures the transition of urgent adaptive reactions of the body to long-term adaptation to loads (Kurpad et al., 2006; Lee \& Shiroma, 2014). A positive effect can be achieved only when the action of one activity is combined with the action of the next, if new activities maintain the sequence of balanced compensation of energy resources (Leonard, 2003; Schorr et al., 2018). In turn, this means that the basis for the development of fitness is the systematic impact of the load and the regularity of its repetition (Exel et al., 2019; Nagovitsyn et al., 2019). Violation of the principle of systematicity and gradualness in the training process can not only not give the desired healing effect, but also lead to serious health problems (Wennman et al., 2019).

In the COVID-19 corona virus pandemic that has developed to date, a significant part of the population of the Russian Federation, Europe and the whole world is in self-isolation in their homes. At home, many citizens of the Russian Federation do not have the opportunity to exercise (Nagovitsyn et al., 2017; Osipov et al., 2016). Thus, violating the basic health principle - the systematic nature of the physical activity of an aerobic nature (Chen et al., 2003; Esliger \& Tremblay, 2007). Today, the Internet offers many programs and methods of physical exercises that encourage users to replace their usual physical activity (Nagovitsyn et al., 2019; Wahl et al., 2017). However, many amateur athletes who have not previously used the Internet fitness industry cannot optimally integrate into the fitness process through a mobile device overnight (Cho \& Tian, 2019; Sushames et al., 2016). Moreover, an individual approach to the implementation of physical activity and a longer period to search for a suitable and accessible type of training are necessary (Iconaru et al., 2018; Nagovitsyn et al., 2018; Yoo et al., 2017). It should be especially noted that many fitness programs on the Internet involve special equipment and facilities, which the majority of the population of the Russian Federation do not have available. In this regard, scientifically based methodological assistance to the population is needed to replace habitual physical exercises with physical activity at home during the spread of COVID-19 in the Russian Federation and around the world.

Despite the identified relevance of the implementation of physical exercises at home, a not sufficiently studied issue is the methodological preparation of student and working youth for independent implementation, selfmonitoring and, on its basis, the development of individual physical activity during the day, week or a specific training cycle. In this regard, the study determined the aim of the study - to develop a system of realization step exercises in home conditions using the independent calorimetry during the period regime of self-isolation and to prove its effectiveness on the indicators of body mass index of the people. 


\section{MATERIAL AND METHODS}

\section{Participants}

62 women and men between the ages of 18 and 45 who have problems with being overweight. All participants in the study during the self-isolation regime did not have special sports equipment for aerobic training at home or in the hostel: a treadmill, a cycling machine, an ellipsoid, a rowing machine, a ladder machine, etc. The study was conducted at home for 60 days. Before the start of the study, all participants in the experiment had the experience of self-study with low and medium intensity of physical activity of an aerobic nature, at least 2-3 times a week. They also possessed the ability to implement self-monitoring of energy exchange of kilocalories with various nutrition and physical activity using mobile devices. Each participant in the experiment received written consent to participate in the study. Prior to the study, the focus group $(n=62)$ was divided into the experimental $(n=30)$ and control $(n=32)$ groups. The experimental group $(E G)$ included participants who, for 60 days, carried out daily physical activity according to the author's system, corresponding to the energy load until the implementation of the self-isolation mode. Participants who implement the daily motor mode without the author's system for the designated time were enrolled in the control group (CG).

\section{Ethics}

The Institute Committee for Medical and Health Research Ethics approved the study (2020/04), which was conducted in accordance with the Helsinki declaration. All results were treated anonymously. Authors conducted their research ethically according to international standards and as required by the journal (Harriss et al., 2019).

\section{Design}

During the experiment, the participants in the EG study, for every day $(n=60)$ of the experiment, it was necessary to realize motor activity, which was supposed to "burn" their consumption of kilocalories during the day. To implement independent physical activity at home, he was offered the following physical exercise step exercise. This physical activity included climbing a sports equipment available at home (step, bench, cube, etc.). The subject became facing the projectile. He independently began the exercise according to the Harvard step test system: put one leg on the shell; then another and straighten up; after that, immediately lower the leg with which the exercise began, in front of the projectile, then the second and return to its original position. The exercise had to be repeated continuously, but not more than 30 minutes. The amount of work power when climbing a projectile and burning kilocalories on it was proposed to be calculated according to the following formula: $X=f^{*} h^{*} W^{*} 0.003$, where "X» is the power of work, kcal/min, "f» is the lifting frequency in 1 minute, «h» is the height projectile in meters, «W» - body weight of the subject, $\mathrm{kg}$.

Based on preliminary research work through the use of the mathematical least-squares method, a formula was developed for an affordable and simple calculation of kilocalories for EG participants. $N=0.00168$ $0.098 / P$, where "N» is the amount of kilocalories burned per 1 heart contraction per 1 kilogram of body weight, «P» is the heart rate during exercise (beats per minute). Using this formula during the experiment, EG participants daily calculated the difference between consumed and "burned" calories per day. The analysis of the daily diet was carried out using food diaries, which they kept for 60 consecutive days.

This period is due to the moment of the announcement of the regime of self-isolation in the Russian Federation to this day. The implementation of the experimental work was focused on targeted stimulation of the participants in the experiment to independent, systematic, optimal motor activity to maintain BMI. The main goal set before the participants of the study before the experiment was to realize physical activity during 
the day that was identical in intensity and energy load, which they realized before the introduction of the selfisolation mode.

To analyse the effect of experimental work on the body of all study participants, express diagnostics was used to determine the level of BMI. BMI formula = weight in kilograms / height in meters squared. Express diagnostics was implemented before the study and after the experimental work.

\section{Statistical analysis}

Statistical processing of research results was carried out using the statistical analysis program SPSS20. The level of reliability of the obtained data was determined using Student's T-test at $p<.05$. Before the experiment, mathematical-statistical uncertainty of the differences between the EG and the CG at $p>.05$ by express diagnostics for determining the BMI level was recorded.

\section{RESULTS}

During the experimental period, the EG participants carried out daily self-monitoring of aerobic motor activity according to a special author's system. The author's system was developed on the basis of analysis of special literature on the study of the energy consumption of respondents for motor activity at different speeds and under different conditions (Brown et al., 2003; Eakin et al., 2007; Sallis \& Saelens, 2000; Troiano et al., 2012). The author's system included a correlation of various types of physical exercises in various speed modes with step exercise in various motor and level modes and the number of kilocalories burned at the corresponding load (Table 1).

Table 1. The author's system for independent monitoring of burned calories under various physical exertion.

\begin{tabular}{|c|c|c|}
\hline Kcal/min * & Exercise characteristic & Step exercise $\left(f^{\star} h\right)^{\star *}$ \\
\hline $0.03333^{*} \mathrm{~W}$ & $\begin{array}{l}\text { Walking }(2.5-3 \mathrm{~km} / \mathrm{h}) \text {; bike simulator }(0.5-0.6 \mathrm{Wat} / \mathrm{kg}) \text {; elementary } \\
\text { gymnastic exercises. }\end{array}$ & $9.5-12$ \\
\hline $0.04167^{*} \mathrm{~W}$ & $\begin{array}{l}\text { Walking }(3-3.5 \mathrm{~km} / \mathrm{h}) \text {; bicycle }(7-8 \mathrm{~km} / \mathrm{h}) \text {; bike simulator }(0.6-0.7 \\
\text { Wat } / \mathrm{kg}) \text {; gymnastic exercises of medium intensity. }\end{array}$ & $12-14.5$ \\
\hline $0.05 * \mathrm{~W}$ & $\begin{array}{l}\text { Walking }(3.5-4 \mathrm{~km} / \mathrm{h}) \text {; bicycle }(8-8.5 \mathrm{~km} / \mathrm{h}) \text {; rowing }(50-55 \mathrm{~m} / \mathrm{min}) \text {; } \\
\text { swimming }(10 \mathrm{~m} / \mathrm{min}) \text {; bike simulator }(0.7-0.8 \mathrm{Wat} / \mathrm{kg}) ; \text { gymnastic } \\
\text { exercises with the intensity above average. }\end{array}$ & $14.5-17$ \\
\hline $0.05833^{*} \mathrm{~W}$ & $\begin{array}{l}\text { Walking }(4.5-5 \mathrm{~km} / \mathrm{h}) \text {; bicycle }(8.5-10 \mathrm{~km} / \mathrm{h}) \text {; swimming }(15 \mathrm{~m} / \mathrm{min}) \text {; } \\
\text { rowing }(55-60 \mathrm{~m} / \mathrm{min}) ; \text { bike simulator }(0.9-1 \text { Wat } / \mathrm{kg}) \text {; sports games with } \\
\text { the average intensity; gymnastic exercises of high-speed. }\end{array}$ & $17-19.5$ \\
\hline $0.06667 * \mathrm{~W}$ & $\begin{array}{l}\text { Walking or slow running }(5.5-6 \mathrm{~km} / \mathrm{h}) \text {; bicycle }(10-15 \mathrm{~km} / \mathrm{h}) \text {; rowing }(60- \\
70 \mathrm{~m} / \mathrm{min}) \text {; swimming }(15-20 \mathrm{~m} / \mathrm{min}) \text {; bike simulator }(1.1-1.2 \text { Wat } / \mathrm{kg}) \text {; } \\
\text { skates or rollers }(8-10 \mathrm{~km} / \mathrm{h}) \text {; sports games with the intensity above } \\
\text { average; dances; gymnastic exercises of speed-power. }\end{array}$ & $19.5-22$ \\
\hline
\end{tabular}

As can be seen from Table 1, the author's system for self-monitoring of burned calories was compiled taking into account the most common physical exertion among the population with free physical activity. Each type of physical activity is differentiated by the intensity of its implementation and corresponds to the implementation of step exercise in the conditions of self-isolation mode at home. During the experiment, the participant in the EG study individually selected the level of physical activity according to his system and his 
previous physical activity before the introduction of the self-isolation mode. This physical activity consisted of a number of relatively independent elements: the physical exercises themselves, preparation for their implementation and outdoor activities. The dosage of each exercise and the sequence of exercises should provide the optimal health-improving dynamics of the heart rate: the maximum during exercise was no more than 150-160 beats/min and the average pulse in the session should not exceed 130-140 beats/min. In these classes, the main criterion for performance, which should be guided by those involved, was continuous monitoring of the body's well-being.

As shown in Figures 1-2, a significant portion of the study participants had a change in BMI before and after the experimental work.
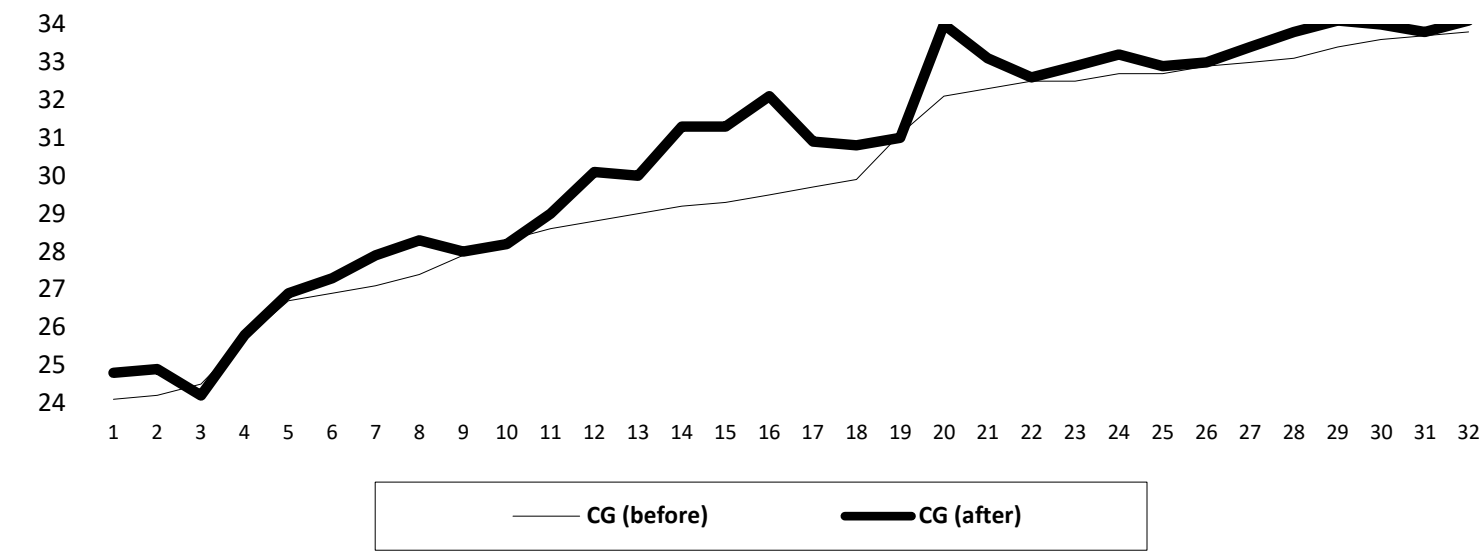

Figure 1. Results of BMI CG before and after the experiment.

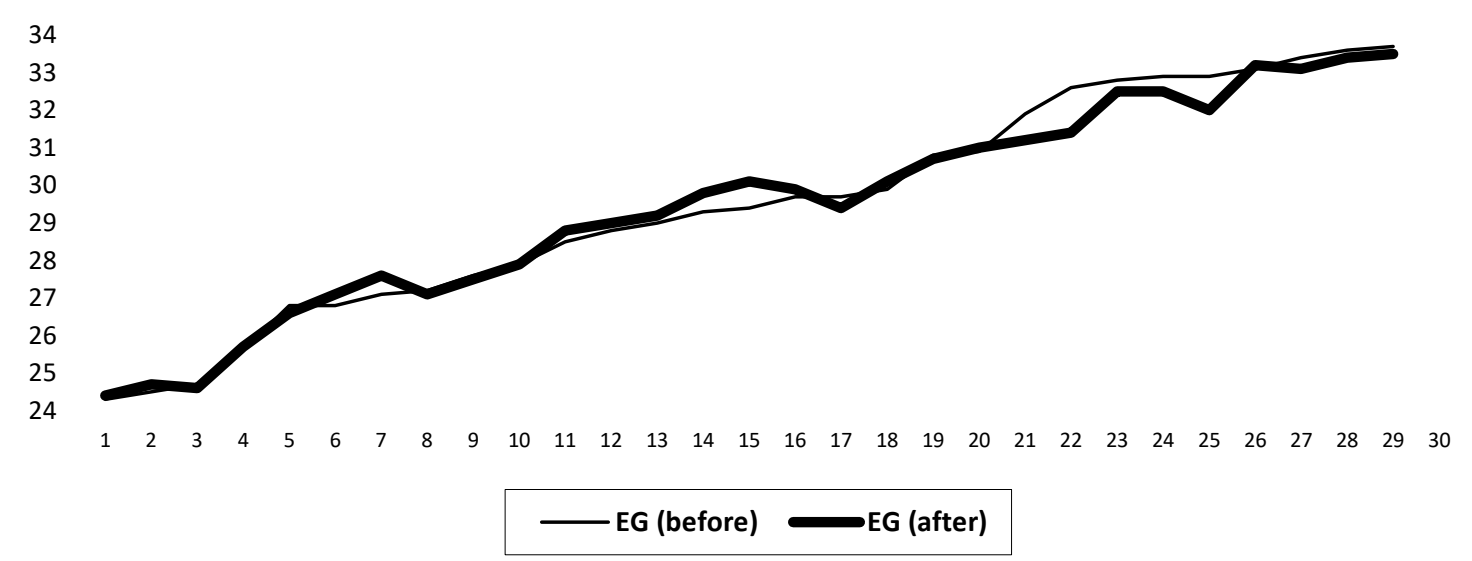

Figure 2. Results of BMI EG before and after the experiment.

The recorded data at the end of the experiment showed the effectiveness of the experimental work. After the experiment, the mathematical-statistical significance of the differences between the EG and the CG at $p<$ .05 was recorded. The participants in the study of CG, compared with EG, significantly increased the level of BMI. Due to the fact that during this time period the body length of the participants could not significantly increase, their body weight significantly increased. This is confirmed by the results of statistical processing 
of CG data before and after the experimental work. The significance of the difference was revealed at $p<$ .05. In turn, the statistical processing of EG data before and after the experimental work showed that the difference was not significant at $p>.05$. From this, the main result of the study can be identified: the implementation of systematic motor activity according to the author's system for self-monitoring of burned calories does not significantly increase the body weight of the participants, having overweight or obesity, in the conditions of implementation of the regime of self-isolation.

\section{DISCUSSION}

The experimentally proven results of this study are consistent with the findings of other studies on the implementation of motor activity of a population of different ages through the introduction of self-monitoring and self-diagnosis technologies (Esliger \& Tremblay, 2007; Osipov et al., 2018; Plotnikoff \& Karunamuni, 2011). Analysis of studies on the dependence of increasing physical health indicators of athletes of various ages, in particular the cardiovascular system (Abbasi, 2019; Schorr et al., 2018; Yarmak et al., 2018), on the increase in their physical activity during a certain fixed cycle proves the reliability and relevance of the results obtained in the study (Hildebrand et al., 2014; Thijs et al., 2019). It is a systematic daily monitoring of aerobic activity that has a positive effect on health-saving energy metabolism in the body.

Numerous studies suggest and experimentally substantiate various approaches to monitoring the motor activity of athletes: from the use of special formulas (Cho \& Tian, 2019; King et al., 2008) to the use of mobile technologies (Henriksen et al., 2018; Wennman, 2019), such as fitness trackers and various types of accelerometers (Lee \& Shiroma, 2014; Yoo et al., 2017). However, as the results of our study showed, only the implementation of self-monitoring through the systematization and classification of the most accessible for young people of various physical exercises of a cyclic nature, allows to achieve a positive result. The dosed load used in the study showed statistically significant results according to the body weight of the subjects. The originality of our study lies in the fact that the study created special recommendations on the expenditure of energy expenditures of respondents for motor activity at different speeds and under different conditions. The systematic monitoring of the implementation of motor activity, proposed in our study, allows us to optimally and consistently adapt the body of the study participant to muscle activity of an aerobic nature in unusual conditions for him to study.

The obtained mathematical and statistical results of this study are associated with the results of research on the experimental proof of the dependence of increased kilocalories burning on the intensity of the load, measured on the basis of calculating the heart rate of the study participants (Kurpad et al., 2006; Leonard, 2003; Westerterp \& Plasqui, 2004). With the introduction of continuous self-monitoring and self-diagnosis technologies, experts reliably determine the effectiveness of various types of motor and energy activity (Freedson \& Miller, 2000; Wahl et al., 2017). A certain dependence of energy consumption on the pulse rate is shown, which can be calculated, and with rather high accuracy (Kashiwazaki, 1999; Luke et al., 1997). As the present study experimentally confirmed, the formula " $N=0.00168-0.098 / P$ " proposed in the study makes it possible to mathematically reliably and comparatively simply calculate the burning of kilocalories for 1 heart contraction per 1 kilogram of body weight of participants in physical exercises with different physical activity.

The results of this study are consistent with the results of other studies on the experimental effectiveness of the self-monitoring process by non-professional athletes using mobile technologies (Henriksen et al., 2018; Nagovitsyn et al., 2019; Wennman et al., 2019). Despite the research data, there are many mobile algorithms that are not confirmed by experimental studies (Iconaru et al., 2018). An overabundance of the proposed mobile applications and methodological recommendations saturates the fitness market with low-quality 
information and algorithms (Chen et al., 2003). An incorrectly constructed continuous monitoring of the state of the student's body has a negative result on its performance (Sushames et al., 2016). A certain distrust of these products is created in society and requires simple and affordable methods of instant verification of the services offered (Lee \& Shiroma, 2014). In this direction, the author's development, based on experimental confirmation in the present study, allows mobile mathematically reliable and relatively simple to carry out calorimetry. The calculation algorithms proposed in the study will allow people to customize mobile technologies and software in accordance with their preferences and needs under the conditions of selfisolation.

\section{CONCLUSIONS}

The author's study supplements previous scientific developments on the implementation of calorimetry for various types of motor activity, including differentiation according to the intensity of physical exercises. The formula " $N=0.00168-0.098 / P$ " experimentally developed in the study allows mathematically reliable and relatively simple calculation of kilocalories burning for various physical activities. In the presented study, for the first time, the regularities of increasing the burning of kilocalories in people at various levels of intensity depending on their body weight were experimentally revealed. The results of the study offer a number of significant recommendations for optimizing the motor activity of students and working youth at home. An original system for the realization of physical activity through the development of special motor activity based on the step exercise, differentiated by different energy modes, is proposed and justified. This approach is highly relevant in solving one of the key problems of youth in the situation of the spread of COVID-19: increasing the number of overweight and obese women and men.

The presented work is limited to a small sample of study participants, covering a separate age period: from 18 to 45 years. The number of participants in the study was heterogeneous by gender. The sample obtained does not make it possible to cover the entire target audience in connection with the self-isolation regime, and also to differentiate groups by male and female sex, since the study was conducted only at the first stage of implementation. Accordingly, the results can be determined as preliminary. For further more detailed analysis, it is necessary to conduct a comparative analysis of a larger sample, differentiated by age, gender groups and levels of physical development. Carrying out such a study will provide more reliable information about the implementation of the calorimetry process of respondents during physical exercises of an aerobic nature.

The study will be useful to a wide range of specialists in the field of physical education and sports, fitness trainers, as well as athletes who are independently engaged in various physical exercises. The results of experimental work on the original method of calorimetry and algorithms for monitoring physical activity may be of interest to developers of mobile applications in the field of health, fitness and sports. The author's recommendations for the implementation of continuous self-monitoring will provide feedback to the user of the physical exercise program, increase motivation for setting individual goals of amateur athletes and strategies for its further achievement. Future research will develop in two main directions. On the one hand, the implementation of performance monitoring to introduce the formula in monitoring the training process of youth of different ages and the duration of physical activity under changing conditions of heart rate. On the other hand, on an experimental search for the most effective level of motor activity for a rational regime of burning calories and restoring the body. An experimental study will cover a larger sample of subjects with different individual capabilities and needs for the implementation of motor activity. 


\section{REFERENCES}

Abbasi, J. (2019). For Mortality, Busting the Myth of 10000 Steps per Day. Jama-Journal of the American Medical Association, 322(6), 492-493. https://doi.org/10.1001/jama.2019.10042

Brown, W., Eakin, E., Mummery, W.K., \& Trost, S. (2003). 10,000 Steps Rockhampton: establishing a multi-strategy physical activity promotion in a community. Health Promot J Austr, 14(2), 95-100. https://doi.org/10.1071/HE03095

Chen, K.Y., Acra, S.A., Majchrzak, K., Donahue, C.L., Baker, L., Clemens, L., Sun, M., \& Buchowski M.S. (2003). Predicting energy expenditure of physical activity using hip- and wrist-worn accelerometers. Diabetes Technol Ther, 5(6), 1023-1033. https://doi.org/10.1089/152091503322641088

Cho, S.J., \& Tian, Y. (2019). Investigating the role of communication between descriptive norms and exercise intentions and behaviors: findings among fitness tracker users. Journal Of American College Health, 29, 1-7. https://doi.org/10.1080/07448481.2019.1679819

Eakin, E.G., Mummery, K., Reeves, M.M., Lawler, S., Schofield, G., Marshall, A., \& Brown, W. (2007). Correlates of pedometer use: results from a community-based physical activity intervention trial (10,000 Steps Rockhampton). Int J Behav Nutr Phys Act, 4, 31-39. https://doi.org/10.1186/1479$\underline{5868-4-31}$

Esliger, D.W., \& Tremblay, M.S. (2007). Physical activity and inactivity profiling: the next generation. Canadian Journal Of Public Health, 98(2), S195-207. https://doi.org/10.1139/H07-107

Exel, J., Mateus, N., Abrantes, C., Leite, N., \& Sampaio, J. (2019). Physical activity and sedentary behavior in amateur sports: master athletes are not free from prolonged sedentary time. Sport Sciences for Health, 15(2), 385-391. https://doi.org/10.1007/s11332-019-00527-3

Freedson, P.S., \& Miller, K. (2000). Objective monitoring of physical activity using motion sensors and $\begin{array}{lllll}\text { heart rate. Res } Q \text { Exerc Sport, } 71(2 \quad \text { Suppl), } & \text { S21-29. }\end{array}$ https://doi.org/10.1080/02701367.2000.11082782

Harriss, D.J., Macsween, A., \& Atkinson, G. (2019). Ethical Standards in Sport and Exercise Science Research: 2020 Update. Int J Sports Med, 40, 813-817. https://doi.org/10.1055/s-0033-1358756

Henriksen, A., Haugen, M.M., Woldaregay, A.Z., Muzny, M., Hartvigsen, G., Hopstock, L.A., \& Grimsgaard, S. (2018). Using fitness trackers and smartwatches to measure physical activity in research: analysis of consumer wrist-worn wearables. J Med Internet Res, 20(3), e110. https://doi.org/10.2196/imir.9157

Hildebrand, M., Van Hees, V.T., Hansen, B.H., \& Ekelund, U. (2014). Age group comparability of raw accelerometer output from wrist-and hip-worn monitors. Medicine and Science in Sports and Exercise, 46(9), 1816-1824. https://doi.org/10.1249/MSS.0000000000000289

Iconaru, E.I., Ciucurel, M., Tudor, M., Georgescu, L. \& Ciucurel, C. (2018). The occupational diary as a smartphone application for monitoring the activities of daily living and physical exertion. Journal of Physical Education and Sport, 18, 2008-2013. https://doi.org/10.7752/jpes.2018.s5298

Kashiwazaki, H. (1999). Heart rate monitoring as a field method for estimating energy expenditure as evaluated by the doubly labeled water method. J Nutr Sci Vitaminol (Tokyo), 45, 79-94. https://doi.org/10.3177/jnsv.45.79

King, A.C., Ahn, D.K., Oliveira, B.M., Atienza, A.A., Castro, C.M., \& Gardner, C.D. (2008). Promoting physical activity through hand-held computer technology. American Journal Of Preventive Medicine, 34(2), 138-142. https://doi.org/10.1016/i.amepre.2007.09.025

Kraus, W.E., Janz, K.F., Powell, K.E., Campbell, W., Jakicic, J., Troiano, R., Sprow, K., Torres, A., \& Piercy, K. (2019). Daily Step Counts for Measuring Physical Activity Exposure and Its Relation to 
Health. Medicine and Science in Sports and Exercise, 51(6), 1206-1212. https://doi.org/10.1249/MSS.0000000000001932

Kurpad, A.V., Raj, R., Maruthy, K.N., Vaz, M. (2006). A simple method of measuring total daily energy expenditure and physical activity level from the heart rate in adult men. Eur J Clin Nutr, 60(1), 32-40. https://doi.org/10.1038/sj.ejcn.1602264

Lee, I.M., \& Shiroma, E.J. (2014). Using accelerometers to measure physical activity in large-scale epidemiological studies: Issues and challenges. British Journal of Sports Medicine, 48(3), 197-201. https://doi.org/10.1136/bjsports-2013-093154

Leonard, W.R. (2003). Measuring human energy expenditure: what have we learned from the flex-heart rate method? Am J Hum Biol, 15(4), 479-489. https://doi.org/10.1002/ajhb.10187

Luke, A., Maki, K.C., Barkey, N., Cooper, R., \& McGee, D. (1997). Simultaneous monitoring of heart rate and motion to assess energy expenditure. Med Sci Sports Exerc, 29(1), 144-148. https://doi.org/10.1097/00005768-199701000-00021

Nagovitsyn, R.S., Bartosh, D.K., Ratsimor, A.Y., \& Neverova, N.V. (2019). Modernization of Regional Continuing Pedagogical Education in the "School-College-Institute. European journal of contemporary education, 8(1), 144-156. https://doi.org/10.13187/ejced.2019.1.144

Nagovitsyn, R.S., Miroshnichenko, A.A., \& Senator, S.Yu. (2018). Implementation of mobile pedagogy during continuous education of physical culture teachers. Integration of Education, 22(1), 107-119. https://doi.org/10.15507/1991-9468.090.022.201801.107-119

Nagovitsyn, R.S., Tutolmin, A.V., Maksimov, Y.G., Dimova, I.A., Karoyan, A.A., Skryabina, D.Y., \& Volkov, S.A. (2019). Motivation for physical activity of people of different ages. Gazzetta Medica Italiana - Archivio per le Scienze Mediche, 178(10), 799-806. https://doi.org/10.23736/S03933660.18.03965-7

Nagovitsyn, R.S., Volkov, P.B. \& Miroshnichenko, A.A. (2017). Planning of physical load of annual cycle of students', practicing cyclic kinds of sports, training. Physical education of students, 21(3), 126133. https://doi.org/10.15561/20755279.2017.0305

Nagovitsyn, R.S., Zekrin, F.H., Fendel', T.V., \& Zubkov, D.A. (2019). Sports selection in martial arts based on the harmonic stability of results at competitions. Journal of Human Sport and Exercise, 14(4proc), S867-S876. https://doi.org/10.14198/ihse.2019.14.Proc4.49

Osipov, A., lermakov, S., Gruzinky, V., Kudryavtsev, M., Bliznevsky, A., Bliznevskaya, V., Serzhanova, Z., Kuzmin, V., Zhavner, T., Vapaeva, A., Markov, K., \& Kondrashova, E. (2018). Analysis of the parameter changes of students' physical development (At the age of 18-20) to identify the threat of increased body weight and obesity. Journal of Physical Education and Sport, 18(2), 800-809. https://doi.org/10.7752/jpes.2018.02118

Osipov, A., Starova, O., Malakhova, A., Vonog, V., Zhavner, T., Salyamova, P., Struchkov, V., Kudryavtsev, M. (2016). Modernization process of physical education of students in the framework of implementation of the state strategy for the development of physical culture, sport and tourism in the Russian Federation. Journal of Physical Education and Sport, 16(4), 1236-1241. https://doi.org/10.7752/jpes.2016.04196

Plotnikoff, R.C., \& Karunamuni, N. (2011). Steps towards permanently increasing physical activity in the population. Curr Opin Psychiatry, 24(2), 162-167. https://doi.org/10.1097/YCO.0b013e3283438107

Sallis, J.F., \& Saelens, B.E. (2000). Assessment of physical activity by self-report: Status, limitations, and future directions. Research Quarterly for Exercise and Sport, 71(sup2), 1-14. https://doi.org/10.1080/02701367.2000.11082780

Schorr, E., Dahl, H., Sarkinen, A., \& Brown, R. (2018). Activity Tracker Increases Daily Step Count PostCardiac Rehabilitation Compared to Placebo Device. Circulation, 137(Suppl_1), AP276. 
Sushames, A., Edwards, A., Thompson, F., McDermott, R., Gebel, K. (2016). Validity and reliability of fitbit flex for step count, moderate to vigorous physical activity and activity energy expenditure. PLoS One, 11(9), e0161224. https://doi.org/10.1371/journal.pone.0161224

Thijs, I., Fresiello, L., Oosterlinck, W., Sinnaeve, P., \& Rega, F. (2019). Assessment of Physical Activity by Wearable Technology During Rehabilitation After Cardiac Surgery: Explorative Prospective Monocentric Observational Cohort Study. JMIR Mhealth Uhealth, 7(1), e9865. https://doi.org/10.2196/mhealth.9865

Troiano, R.P., Pettee, G., Welk, G.J., Owen, N., Sternfeld, B. (2012). Reported physical activity and sedentary behavior: why do you ask? Journal of physical activity \& health, 9(1), S68-75. https://doi.org/10.1123/ipah.9.s1.s68

Wahl, Y., Düking, P., Droszez, A., Wahl, P., \& Mester, J. (2017). Criterion-validity of commercially available physical activity tracker to estimate step count, covered distance and energy expenditure during sports conditions. Front Physiol, 8, 725-730. https://doi.org/10.3389/fphys.2017.00725

Wennman, H., Pietilä, A., Rissanen,H., Valkeinen, H., Partonen, T., Mäki-Opas, T., \& Borodulin, K. (2019). Gender, age and socioeconomic variation in 24-hour physical activity by wrist-worn accelerometers: the FinHealth 2017 Survey. Scientific Reports, 9(1), 6534. https://doi.org/10.1038/s41598-019-43007-x

Westerterp, K., \& Plasqui, G. (2004). Physical activity and human energy expenditure. Curr Opin Clin Nutr Metab Care, 7(6), 607-613. https://doi.org/10.1097/00075197-200411000-00004

Yagotin, R.S., Degtyarenko, T.V., Bosenko, A.I., Plisko, V. \& Dolinsky, B. (2019). Criterion score of the physical and psychophysiological condition of students in the context of determining their individual adaptability to physical loads. Physical education of students, 23(1), 51-57. https://doi.org/10.15561/20755279.2019.0108

Yarmak, O., Blagii, O., Palichuk, Y., Hakman, A., Balatska, L., Moroz, O., \& Galan, Y. (2018). Analysis of the factor structure of the physical condition of girls 17-19 year-old. Journal of Human Sport and Exercise, 13(2proc), S259-S268. https://doi.org/10.14198/ihse.2018.13.Proc2.11

Yoo, D.H., Ko, D.S., \& Yeo, I.S. (2017). Effect of user's trust in usefulness, attitude and intention for mobile sports content services. Journal of Physical Education and Sport. 17(1), 92-96. https://doi.org/10.7752/jpes.2017.s1014 